\title{
Reduced-Complexity Maximum-Likelihood Detection in Downlink SDMA Systems
}

\author{
Jos Akhtman, Chun-Yi Wei and Lajos Hanzo \\ School of ECS., Univ. of Southampton, SO17 1BJ, UK. \\ Tel: +44-23-80-593 125, Fax: +44-23-80-593 045 \\ Email: lh@ecs.soton.ac.uk, http://www-mobile.ecs.soton.ac.uk
}

\begin{abstract}
The literature of up-link SDMA systems is rich, but at the time of writing there is a paucity of information on the employment of SDMA techniques in the down-link. Hence, in this paper a Space Division Multiple Access (SDMA) down-link (DL) multi-user communication system invoking a novel low-complexity Maximum Likelihood (ML) space-time detection technique is proposed, which can be regarded as an advanced extension of the Complex Sphere Decoder (CSD). We demonstrate that as opposed to the previously published variants of the CSD, the proposed technique may be employed for obtaining a high effective throughput in the so-called "over-loaded" scenario, where the number of transmit antennas exceeds that of the receive antennas. The proposed method achieves the optimum performance of the ML detector even in heavily over-loaded scenarios, while the associated computational complexity is only moderately increased. As an illustrative example, the required $E b / N O$ increased from $2 \mathrm{~dB}$ to $9 \mathbf{d B}$, when increasing the normalized system load from unity, representing the fully loaded system, to a normalized load of $\mathbf{1 . 5 5 6}$.
\end{abstract}

\section{INTRODUCTION}

Multiple Input and Multiple Output (MIMO) systems employing multiple antennas at both the transmitter and receiver exhibit a substantially higher spectral efficiency than conventional single-antenna systems. The flexible configuration of a MIMO system's antennas allows us to satisfy a number of potentially contradictory design objectives in terms of the achievable multiplexing and diversity gain, hence this topic has recently attracted substantial research attention [1], [2]. Space Division Multiple Access (SDMA) constitutes an attractive MIMO subclass, which is capable of achieving a high user capacity by supporting a multiplicity of subscribers within the same frequency bandwidth [3], [4] ${ }^{1}$.

The efficient design of the down-link transmitter is of paramount importance for the sake of achieving a high throughput. The effects of Multi-User Interference (MUI) may be mitigated by employing spatio-temporal pre-proccessing at the transmitter. Consequently, the down-link receiver's complexity may be reduced with the advent of transmit pre-processing at the base station, a technique, which is also often referred as Multi-User Transmission (MUT) [7].

Furthermore, in the context of MUT often Time Division Duplexing (TDD) is invoked for separating the up- and down-link traffic. This is, because when the Channel's Impulse Response (CIR) may be assumed to be both quasi-stationary and similar in the up- and downlink, we may assume that the channel transfer function estimated in the up-link may be used for spatio-temporal preprocessing in the down-link. A witty approach to spatio-temporal preproccessing was proposed by Vandenameele et. al. in [4], where the MUT transformation matrix was specifically designed so that its product

Acknowledgements: The work reported in this paper has formed part of the Wireless Enabling Techniques work area of the Core 3 Research Programme of the Virtual Centre of Excellence in Mobile and Personal Communications, Mobile VCE, www.mobilevce.com, whose funding support, including that of EPSRC, is gratefully acknowledged. Fully detailed technical reports on this research are available to Industrial Members of Mobile VCE.

${ }^{1}$ Other MIMO systems, such as beamformers [5] and space-time codes [6] are not considered in this contribution. with the channel matrix yielded an identity matrix. This MUT transformation matrix may be regarded as a perfect pre-equalizer, which effectively results in a MUI-free channel. Furthermore, Choi and Murch [8] proposed an attractive precoder design, which allows for a specific user to receive his/her dedicated signal, entirely free from MUI inflicted by other users. A somewhat similar preprocessing method, which is referred to as the Block Diagonalization Algorithm (BDA), was discussed in [9], which relied on employing the Singular Value Decomposition (SVD). More specifically, the spatio-temporal pre-processing technique of [8] decomposes a MIMO channel into a set of parallel single-user MIMO channels, which facilitates the employment of well-known MIMO-processing techniques [3], [4].

For the sake of increasing the effective system throughput, we consider a so-called overloaded scenario, where we have a higher number of antennas in the transmitter than that in the receiver. Furthermore, we advocate a MUT technique, which is capable of reliable detection in this overloaded system. However, a powerful detection algorithm is required at the receiver, in order to support the high-integrity operation of our overloaded system configuration. The performance of the family of classic linear detectors, such as the Minimum Mean Square Estimation (MMSE) detector was shown to be unsatisfactory in the overloaded scenario [10]. Consequently, we invoke a nonlinear detector. However, the typically high complexity of nonlinear detectors is often prohibitive in practical systems. Thus, a Reduced Search Algorithm (RSA) may be employed for reducing the complexity of the nonlinear detector. In this contribution a novel Optimized Hierarchy $(\mathrm{OH})$ RSA-aided ML detection method is advocated [11], which may be regarded as an advanced extension of the CSD techniques portrayed in [12]. As opposed to the CSD, the OHRSA considered exhibits a relatively low complexity even in heavily overloaded scenarios and thus its employment is meritorious. Finally, we introduce an objective measure of the system's achievable throughput in support of our performance-related discussions.

The rest of this paper is structured as follows. In Section II we outline the system model used, while in Section III our system throughput measure is introduced. The reduced-complexity maximum-likelihood detection algorithm advocated is summarized in Section IV, along with the portrayal of our soft-bit information generation technique used for invoking channel coding. Out performance results are provided in Section VI. Finally, we conclude our discourse in Section VII.

\section{SYSTEM MODEL}

The DL-SDMA system considered is depicted in Figure 1. More specifically, our system comprises a Base-Station (BS) employing $M$ transmit antennas as well as $K$ Mobile Station (MS) each employing $N_{k}$ receive antennas.

In this contribution we consider a flat fading MIMO channel. Consequently, each link between the $i$-th BS transmit antenna and the $j$-th MS receiver antenna of the $k$-th user may be characterized by a complex-valued scalar channel coefficient $H_{i j}^{(k)}$, which we assume to 


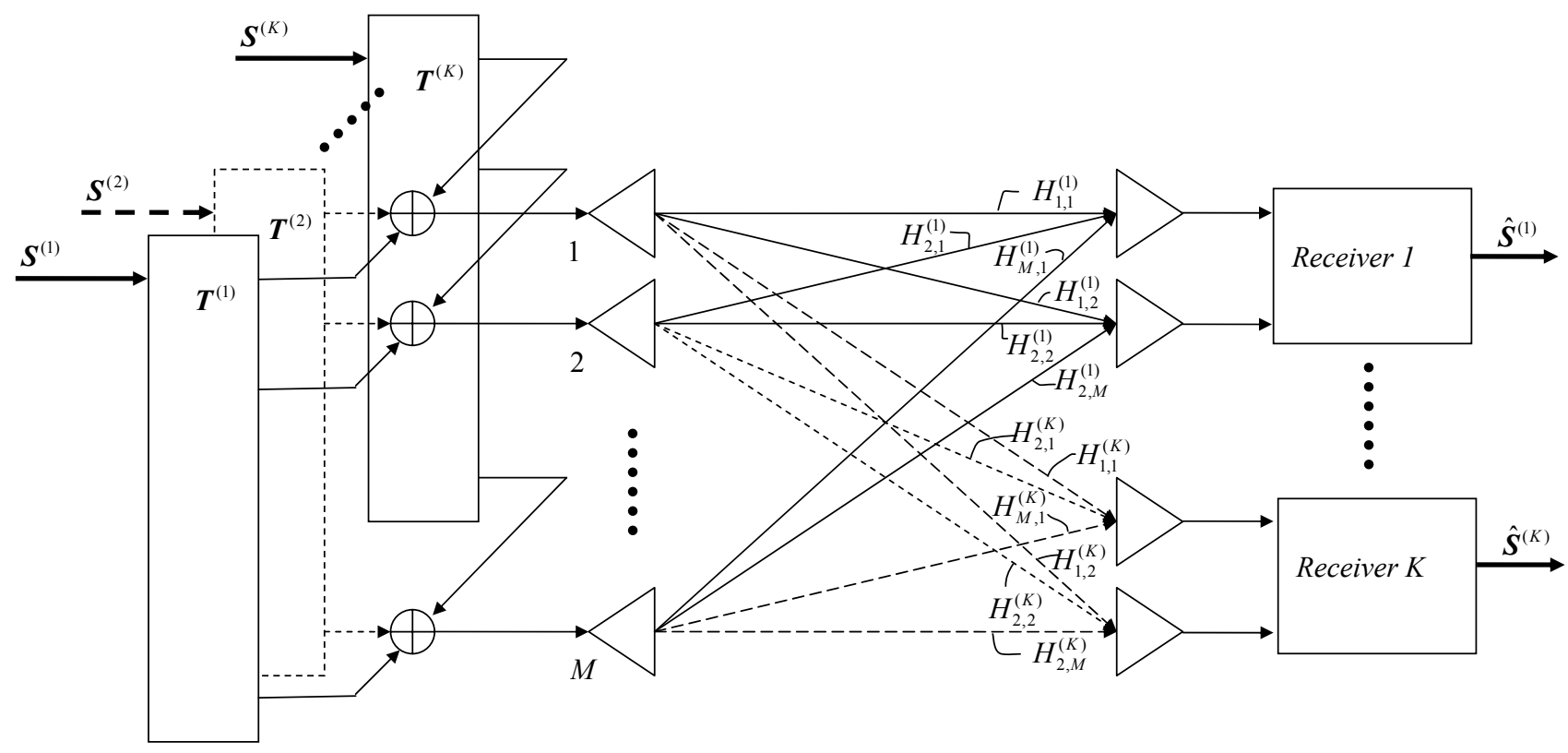

Base Station

Fig. 1. DownLink SDMA System

be an i.i.d. Gaussian random variable having a variance of unity and a mean of zero. Moreover, the MIMO channel corresponding to the $k$-th user may be described as an $\left(N_{k} \times M\right)$-dimensional complexvalued time-domain channel matrix $\mathbf{H}^{(k)}$, which may be defined as follows

$$
\mathbf{H}^{(k)}=\left(\begin{array}{cccc}
H_{1,1}^{(k)} & H_{1,2}^{(k)} & \cdots & H_{1, M}^{(k)} \\
H_{2,1}^{(k)} & H_{2,2}^{(k)} & \cdots & H_{2, M}^{(k)} \\
\vdots & \vdots & \cdots & \vdots \\
H_{N_{k}, 1}^{(k)} & H_{N_{k}, 2}^{(k)} & \cdots & H_{N_{k}, M}^{(k)}
\end{array}\right) .
$$

Finally, the entire multi-user MIMO channel may be characterized by the supermatrix $\mathbf{H}$, which may be constructed by concatenating the corresponding channel matrices associated with each of the MSs. Thus we have

$$
\mathbf{H}=\left(\begin{array}{llll}
\mathbf{H}^{(1)} & \mathbf{H}^{(2)} & \cdots & \mathbf{H}^{(K)}
\end{array}\right)^{T} .
$$

Let $\mathbf{s}^{(k)} \in \mathbb{C}^{L_{k} \times 1}$ be a complex-valued column vector, which denotes the data symbol vector to be transmitted for the $k$-th MS. Furthermore, let $\mathbf{r}^{(k)}$ and $\mathbf{n}^{(k)}$ be the received signal vector and noise vector associated with the $k$-th MS, respectively. Additionally, we define a so-called space-time preprocessor matrix $\mathbf{T}^{(k)} \in \mathbb{C}^{N_{k} \times L_{k}}$, which was designed for the sake of eliminating the MUI [8]. The composite multiuser received signal encountered by all receivers may be formulated as a column-based supervector constituted by the received signal vectors associated with each of the user terminals. Thus we have

$$
\left(\begin{array}{c}
\mathbf{r}^{(1)} \\
\mathbf{r}^{(2)} \\
\vdots \\
\mathbf{r}^{(K)}
\end{array}\right)=\mathbf{H T}\left(\begin{array}{c}
\mathbf{s}^{(1)} \\
\mathbf{s}^{(2)} \\
\vdots \\
\mathbf{s}^{(K)}
\end{array}\right)+\mathbf{n}
$$

where

$$
\mathbf{T}=\left(\begin{array}{llll}
\mathbf{T}^{(1)} & \mathbf{T}^{(2)} & \ldots & \mathbf{T}^{(K)}
\end{array}\right)
$$

and

$$
\mathbf{n}=\left(\begin{array}{llll}
\mathbf{n}^{(1)} & \mathbf{n}^{(2)} & \cdots & \mathbf{n}^{(K)}
\end{array}\right)^{T}
$$

are the space-time preprocessor's supermatrix, as well as the AWGN noise supervector constructed by concatenating the corresponding quantities associated with each of the user terminals. Observe that Figure 1 illustrates a particular scenario, where each of the user terminals employs two receive antennas.

To elaborate a little further, we may express the effective channel supermatrix HT of Equation (3) as follows

$$
\mathbf{H} \mathbf{T}=\left(\begin{array}{ccccc}
\mathbf{H}^{(1)} \mathbf{T}^{(1)} & \ldots & \mathbf{H}^{(1)} \mathbf{T}^{(k)} & \ldots & \mathbf{H}^{(1)} \mathbf{T}^{(K)} \\
\vdots & \ldots & \vdots & \ldots & \vdots \\
\mathbf{H}^{(k)} \mathbf{T}^{(1)} & \ldots & \mathbf{H}^{(k)} \mathbf{T}^{(k)} & \ldots & \mathbf{H}^{(k)} \mathbf{T}^{(K)} \\
\vdots & \ldots & \vdots & \ldots & \vdots \\
\mathbf{H}^{(K)} \mathbf{T}^{(1)} & \ldots & \mathbf{H}^{(K)} \mathbf{T}^{(k)} & \ldots & \mathbf{H}^{(K)} \mathbf{T}^{(K)}
\end{array}\right) .
$$

Observe that resolving the MUI expressed in Equation (3) is equivalent to separating the system model of Equation (3) into a set of single user-related expressions of the following form

$$
\mathbf{r}^{(k)}=\mathbf{H}^{(k)} \mathbf{T}^{(k)} \mathbf{s}^{(k)}+\mathbf{n}^{(k)},
$$

where the $\left(N_{k} \times L_{k}\right)$-dimensional matrix $\mathbf{H}^{(k)} \mathbf{T}^{(k)}$ characterizes the effective channel cooresponding to the $k$-th MS.

Consequently, we are aiming for constructing a MUT preprocessor $\mathbf{T}$, so that we arrive at a diagonal supermatrix $\mathbf{H T}$ in the form of Equation (6), which has non-zero matrix-elements $\mathbf{H}^{(k)} \mathbf{T}^{(k)}$ along its main diagonal and zero matrix-elements $\mathbf{H}^{(i)} \mathbf{T}^{(j)}, i \neq j$ elsewhere. More specifically, we have the following MUT preprocessor design criterion

$$
\mathbf{H}^{(i)} \mathbf{T}^{(k)}=\mathbf{0}, i \neq k, i, k=1, \ldots, K .
$$

As it was demonstrated in [8], the $n$-th column of the MUT preprocessor $\mathbf{T}^{(k)}$, which we denote as $t_{n}^{(k)}$, has to be in the null 
space of $\mathbf{H}^{(i)}, i \neq k$. More explicitly, we have [8]

$$
t_{n}^{(k)} \in \bigcap_{i=1, i \neq k}^{K} \operatorname{ker}\left(\mathbf{H}^{(i)}\right), \quad \text { for } k=1,2, \cdots, K,
$$

where $\operatorname{ker}(\mathbf{X})$ denotes the null space or kernel of the matrix $\mathbf{X}$, while $\bigcap_{i=1, i \neq k}^{K} Y$ is the intersection of the sets $Y_{i}, i=1,2, \cdots$.

Let $\mathbf{V}^{(k)}$ denote an $\left(M \times n_{k}\right)$-dimensional matrix representing an orthogonal basis of the subspace $\bigcap_{i=1, i \neq k}^{K} \operatorname{ker}\left(\mathbf{H}^{(i)}\right)$, where $n_{k}$ is the dimension of this subspace. As suggested by Choi and Murch in [8], we may formulate a solution of the MUT preprocessor design problem as $\mathbf{T}^{(k)}=\mathbf{V}^{(k)} \mathbf{A}^{(k)}$, where $\mathbf{A}^{(k)}$ is a nonzero $\left(n_{k} \times L_{k}\right)$ dimensional matrix. Subsequently, the design of the matrices $\mathbf{V}^{(k)}$ and $\mathbf{A}^{(k)}$ may be summarized in the following three steps.

Step 1: Let us define the quantity $\tilde{\mathbf{H}}^{(k)}$ by omitting the $k$-th matrix-element from the supermatrix $\mathbf{H}$ of Equation (2) describing the channels. Thus, we have

$$
\tilde{\mathbf{H}}^{(k)}=\left(\begin{array}{llllll}
\mathbf{H}^{(1)} & \ldots & \mathbf{H}^{(k-1)} & \mathbf{H}^{(k+1)} & \ldots & \mathbf{H}^{(K)}
\end{array}\right)^{T} .
$$

The null space basis $\mathbf{V}^{(k)}$ can be calculated using the SVD [13] of $\tilde{\mathbf{H}}^{(k)}$ expressed as:

$$
\tilde{\mathbf{H}}^{(k)}=\left(\begin{array}{cc}
\tilde{\mathbf{U}}^{(k)} & \mathbf{U}^{(k)}
\end{array}\right) \cdot\left(\begin{array}{cc}
\sum_{0} & \mathbf{0} \\
\mathbf{0} & \mathbf{0}
\end{array}\right) \cdot\left(\begin{array}{c}
\tilde{\mathbf{V}}^{(k)^{H}} \\
\mathbf{V}^{(k)^{H}}
\end{array}\right) .
$$

Step 2: The specific choice of the $\left(n_{k} \times L_{k}\right)$-dimensional matrix $\mathbf{A}^{(k)}$ in $\mathbf{T}^{(k)}=\mathbf{V}^{(k)} \mathbf{A}^{(k)}$ may depend on a particular system design. For instance, in the simple scenario considered in this paper, where we have $n_{k}=L_{k}$, we may choose $\mathbf{A}^{(k)}$ to be a scaled identity matrix. As an example of an alternative solution, consider the somewhat more sophisticated system design described in [14]. More specifically, the system outlined in [14] employs pre-filters at the transmitter and post-filters at all the receivers, in order to achieve a near-capacity throughput. Correspondingly, the matrix $\mathbf{A}^{(k)}$ was chosen as the right-hand-side SVD factor of the matrix $\mathbf{H}^{(k)} \mathbf{V}^{(k)}$ [14].

Step 3: Calculate $\mathbf{T}^{(k)}=\mathbf{V}^{(k)} \mathbf{A}^{(k)}$ according to the results obtained in Steps 1 and 2.

Note that according to Equation (11) the nonzero matrices $\mathbf{T}^{(k)}$ exist only in the scenario when $\tilde{\mathbf{H}}^{(k)}$ has more columns than rows. Consequently, we have to satisfy the following condition

$$
M>\max \left\{\sum_{i=1, i \neq k}^{K} N_{i}, k=1,2, \ldots, K\right\} .
$$

Moreover, the rank $n_{k}$ of the null space basis $\mathbf{V}^{(k)}$ may be expressed as

$$
n_{k}=M-\sum_{i=1, i \neq k}^{K} N_{i} .
$$

Observe that the particular value of $n_{k}$ will directly affect the spatial multiplexing gain achievable by the system. The subject of the achievable multiplexing gain as well as transmit diversity gain will be further explored in the next section.

\section{Spatial Multiplexing AND TRAnsmit Diversity}

The DL-SDMA system considered in this contribution provides a flexible system design framework capable of supporting various spatial multiplexing and diversity requirements. More specifically, an equivalent single-user SDMA system may potentially provide a spatial multiplexing gain of factor $\min \left\{n_{k}, N_{k}\right\}$ [1]. Furthermore, in the scenario of having a $K$-user SDMA system, where the corresponding MIMO channel may be decomposed into $K$ number of parallel single-user MIMO channels, the system may potentially achieve a spatial multiplexing gain, which increases linearly with the value of $\min \left\{K n_{k}, K N_{k}\right\}$.

As stated above, in this contribution we considered a particular scenario of having $L_{k}=n_{k}$, where the corresponding dimension of the effective transmit antenna array encountered by the $k$-th MS is $L_{k}$. Consequently, the achievable spatial multiplexing gain will increase linearly with the value of $\min \left\{K L_{k}, K N_{k}\right\}$. Furthermore, the achievable transmit diversity increases with the number of physical transmit antennas, $M$. Observe that the MUT's preprocessor matrix $\mathbf{T}^{(k)}$ transforms the signal vector $\mathbf{s}^{(k)}$ into the column space of $\mathbf{T}^{(k)}$. Hence, each element of $\mathbf{s}^{(k)}$ contributes to each of the signals transmitted from the corresponding physical transmit antennas. For instance, let $x_{i}$ denote the symbol transmitted from the $i$-th transmit antenna. Then $x_{i}, i=1, \cdots, M$, comprises contributions from each element of the transmit symbol vector $\mathbf{s}^{(k)}$, which results in the transmit diversity gain achievable by the system.

In the scenario of having an $(M \times N)$-dimensional MIMO channel, where $M$ and $N$ refer to the number of transmit antennas as well as to the total number of antennas employed by all user terminals, different system configurations of $M$ and $N$ provide different spatial multiplexing and transmit diversity gains. In order to categorize the potential system design options relevant to the current discussion, let us introduce the measure of the normalized system load expressed as

$$
L_{s}=\frac{M}{N} \text {. }
$$

Consequently, we may distinguish three different scenarios as follows:

1) under-loaded scenario, for $L_{s}<1$;

2) fully-loaded scenario, for $L_{s}=1$;

3) over-loaded scenario, for $L_{s}>1$.

In the under-loaded case, the number of the antennas in the receiver exceeds that in the transmitter. Hence, the extra receiver antennas may potentially provide the system with the corresponding receive diversity. On the other hand, in the fully-loaded case, the receiver antennas provide a degree of freedom, which is just sufficient for a linear detector to separate the $M$ independent users' signals. Finally, in the over-loaded case, the number of receiver antennas is insufficient for providing an adequate degree of freedom required by a linear detector. Thus, non-linear detection techniques have to be employed. The normalized system load $L_{s}$ of Equation (14) may be utilized in order to characterize both up- and down-link SDMA systems.

\section{Optimized Hierarchy Reduced Search Algorithm Aided MAXimum LiKelihood Detection}

In this section we outline the principles of the Optimized Hierarchy Reduced Search Algorithm (OHRSA) [11], which we invoked in our DS-SDMA system in order to reduce the computational complexity imposed by the Maximum Likelihood (ML) detector employed by each of the MSs. For the sake of convenience, in our forthcoming discussions, we will focus on a single MS and omit the user index $k$. The conventional ML solution is given by [3]

$$
\hat{\mathbf{s}}=\arg \min _{\breve{\mathbf{s}} \in \mathcal{M}^{L}}\|\mathbf{U}(\breve{\mathbf{s}}-\hat{\mathbf{x}})\|^{2},
$$

where $\mathcal{M}$ denotes the constellation size of the modulation scheme employed and $\mathcal{M}^{L}$ is the total set of legitimate values hosted by the transmitted symbol vector $\breve{\mathbf{s}}$. Moreover, $\mathbf{U}$ is an uppertriangular matrix having real-valued elements on its main diagonal and satisfying

$$
\mathbf{U}^{H} \mathbf{U}=\left(\mathbf{H}_{e}{ }^{H} \mathbf{H}_{e}+\sigma_{n}^{2} \mathbf{I}\right),
$$


while

$$
\hat{\mathbf{x}}=\left(\mathbf{H}_{e}{ }^{H} \mathbf{H}_{e}+\sigma_{n}^{2} \mathbf{I}\right)^{-1} \mathbf{H}_{e}{ }^{H} \mathbf{r},
$$

and the effective channel matrix is given by $\mathbf{H}_{e}=\mathbf{H} \cdot \mathbf{T}$, where again, we omit the user index $k$ for the sake of brevity. Consequently, let us define the following objective function [11]

$$
\begin{aligned}
\mathcal{J}(\breve{\mathbf{s}}) & =\|\mathbf{U}(\breve{\mathbf{s}}-\hat{\mathbf{x}})\|^{2}=(\breve{\mathbf{s}}-\hat{\mathbf{x}}) \mathbf{U}^{H} \mathbf{U}(\breve{\mathbf{s}}-\hat{\mathbf{x}}), \\
& =\sum_{i=1}^{L}\left|\sum_{j=i}^{L} u_{i j}\left(\breve{s}_{j}-\hat{x}_{j}\right)\right|^{2}=\sum_{i=1}^{L} \phi_{i}\left(\breve{\mathbf{s}}_{i}\right), \\
\mathcal{J}_{i}\left(\breve{\mathbf{s}}_{i}\right) & =\mathcal{J}_{i+1}\left(\mathbf{s}_{i+1}\right)+\phi_{i}\left(\breve{\mathbf{s}}_{i}\right), \quad i=1, \cdots, L,
\end{aligned}
$$

where we have $\breve{\mathbf{s}}=\left(\breve{s}_{i}, \cdots, \breve{s}_{L}\right)$ and

$$
\mathcal{J}(\breve{\mathbf{s}})=\mathcal{J}_{1}\left(\breve{\mathbf{s}_{1}}\right)>\mathcal{J}_{2}\left(\breve{\mathbf{s}_{2}}\right)>\cdots>\mathcal{J}_{L}\left(\breve{\mathbf{s}_{L}}\right)>0 .
$$

Equation (19) and (20) enable us to employ a highly efficient reduced-complexity search algorithm, which decreases the number of objective function evaluations associated with solving the minimization problem of Equation (15) to a small fraction of the entire set $\mathcal{M}^{L}$

\section{Generation of Soft-Bit Information}

It is widely recognized [3] that the BER associated with the process of communicating over a noisy fading MIMO channel can be dramatically reduced by means of employing channel coding. A particularly effective channel coding scheme is constituted by the softinput soft-output turbo coding technique [6]. Turbo coding, however, requires soft information concerning the bit decisions at the output of the detector, in other words the a posteriori soft information regarding the confidence of the bit-decision is required.

The derivation of an expression for the low-complexity evaluation of the soft-bit information associated with the bit estimates of the detector's output characterized by Equation (15) is given in [11]. Specifically, it is demonstrated in [11] that the soft-bit value associated with the $m$ th bit of the $i$ th QAM symbol of the data symbol vector assigned to the $k$ th user may be closely approximated as

$$
L_{i m}^{(k)} \approx \frac{1}{\sigma_{w}^{2}}\left[\mathcal{J}\left(\breve{\mathbf{s}}_{i m ; \min }^{0}\right)-\mathcal{J}\left(\breve{\mathbf{s}}_{i m ; \min }^{1}\right)\right],
$$

where both $\check{\mathbf{s}}_{i m ; \text { min }}^{b}$ and the corresponding cost function value $J\left(\check{\mathbf{s}}_{i m ; \text { min }}^{b}\right)$ may be obtained by applying the extended OHRSAaided search derived in [11] and briefly discussed in Section IV. The resultant soft-output OHRSA method, which we refer to as the Soft OPtimized HIErarchy (SOPHIE) algorithm exhibits a near-Log-MAP performance, while requiring a dramatically reduced computational complexity, as we demonstrate in the following section.

\section{Simulation Results}

In this section, we characterize the achievable performance of the DL-SDMA system employing the proposed SOPHIE detector. Our simulations were performed in the discrete base-band domain. We employed 4-QAM modulation protected by rate-1/2 turbo coding using a 1000-bit interleaver. All system configurations considered supported $K=3$ users. Finally, for the sake of convenience, we assume $L_{1}=L_{2}=\cdots=L_{K}=L$.

Figure 2 portrays our performance results corresponding to two different system load scenarios, namely to $L_{s}=1.0$ and 1.5. The system configuration and the corresponding normalized system loads are detailed in Table I. For each system load scenario, we characterize the BER performance exhibited by the SOPHIE detector along with

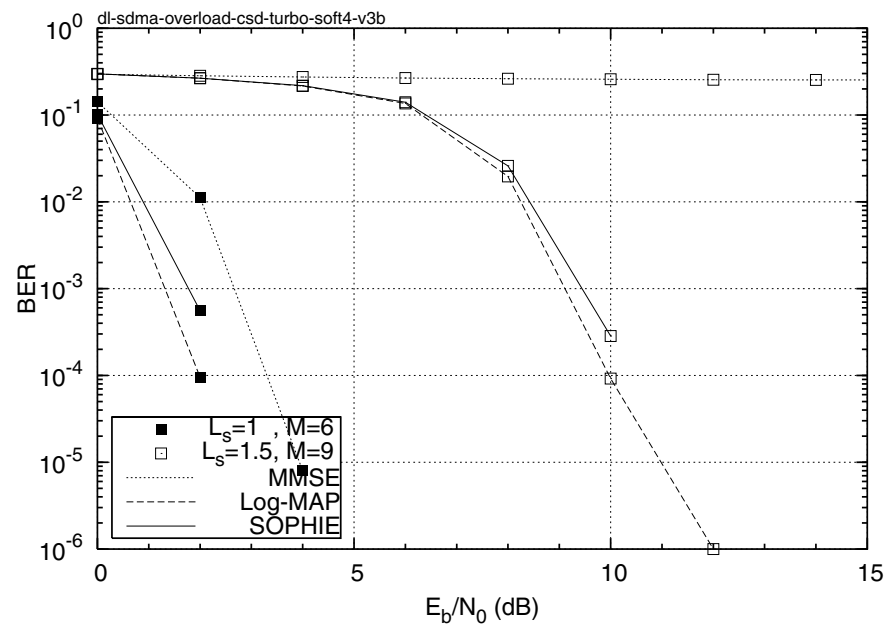

Fig. 2. BER performance of the 4QAM-modulated DL-SDMA scheme having a normalized system load of $L_{s}=1.0$ and 1.5. Our system supports $K=3$ users, where each user employs $N_{k}=2$ receives antennas.

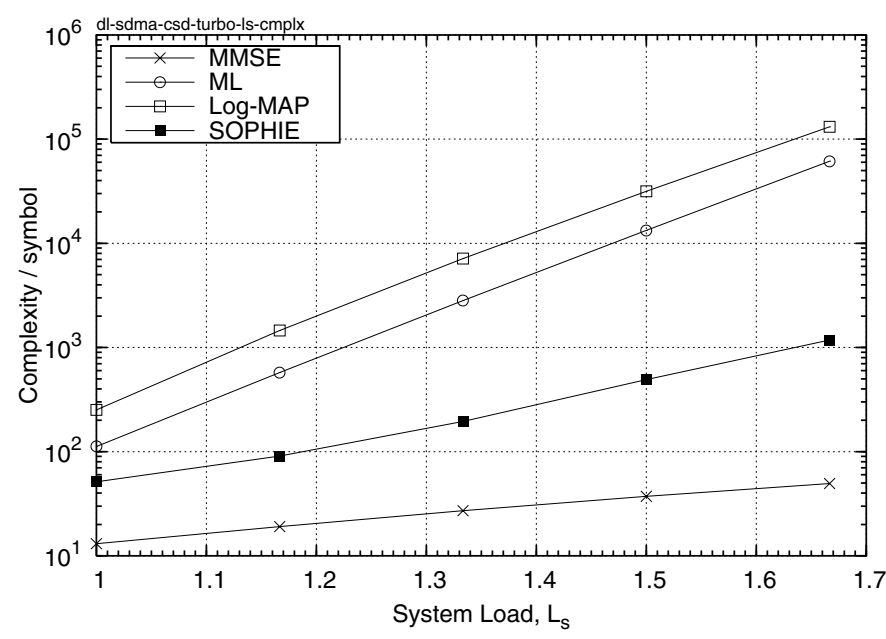

Fig. 3. Complexity comparison of the 4QAM-modulated DL-SDMA scheme having different normalized loads at an SNR of $6 \mathrm{~dB}$. The normalized system load $L_{s}$ ranging from 1 to 1.667 corresponds to the system configuration (A) of Table I supporting $K=3$ users with the aid of $N_{k}=2$ antennas at each receiver.

\begin{tabular}{|c|c|c|c|c|c|}
\hline$M$ & 6 & 7 & 8 & 9 & 10 \\
\hline$L$ & 2 & 3 & 4 & 5 & 6 \\
\hline$L_{s}$ & 1 & 1.167 & 1.333 & 1.5 & 1.667 \\
\hline \multicolumn{7}{|c|}{ TABLE I }
\end{tabular}

SYSTEM CONFIGURATION (A)

\begin{tabular}{|c|c|c|c|c|c|c|}
\hline$M$ & 9 & 10 & 11 & 12 & 13 & 14 \\
\hline$L$ & 3 & 4 & 5 & 6 & 7 & 8 \\
\hline$L_{s}$ & 1 & 1.111 & 1.222 & 1.333 & 1.444 & 1.556 \\
\hline
\end{tabular}

TABLE II

SYSTEM CONFIGURATION (B) 


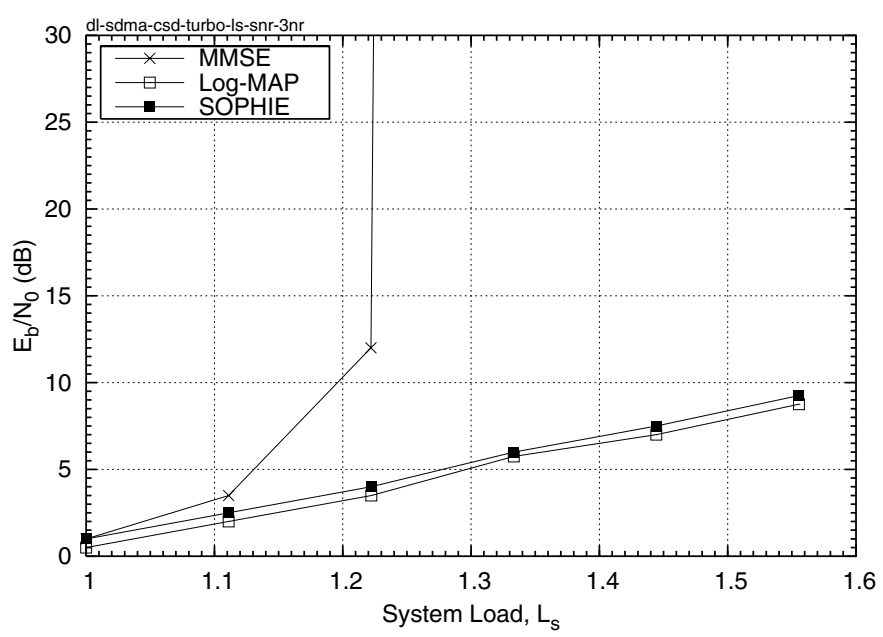

Fig. 4. The required SNR for a target BER=10e-4 with different system load scenarios. System load $L_{s}$ ranging from 1 to 1.556 corresponds to system configuration (B) of Table II with fixed numbers $K=3$ of users and $N_{k}=3$ of antennas at each receiver.

the corresponding BER performances exhibited by both the MMSE and the log-MAP detectors as benchmarkers. In the scenario of $L_{s}=1.0$ all three detectors exhibit an adequate performance. However, when the normalized system load $L_{s}$ is increased to 1.5, which corresponds to a highly overloaded scenario, the MMSE detector fails to attain a satisfactory BER performance. The SOPHIE detector, on the other hand, performs well and the corresponding BER performance is fairly close to that exhibited by the exhaustive search aided log-MAP detector.

Figure 3 characterizes the computational complexity imposed by the different detectors considered. The corresponding system configuration and normalized system load characteristics are detailed in Table I. The associated complexity is quantified in Figure 3 in terms of the total number of additions and multiplications per detected QAM symbol as a function of the system load $L_{s}$ recorded at $\mathrm{SNR}=6 \mathrm{~dB}$. The complexity figures associated with the exhaustive search-based ML and log-MAP are also provided for the sake of comparison. Observe that the SOPHIE detector exhibits a complexity, which is two orders of magnitude lower than that imposed by the logMAP detector, while their performance is quite similar. Moreover, the efficiency of the SOPHIE detector becomes more obvious upon increasing the system load.

The SNR required by the different systems for the sake of achieving a target BER of $10^{-4}$ is quantified in Figure 4 as a function of the system load $L_{s}$. The associated system configuration and normalized system load are detailed in Table II. As we can see in Figure 4, the SNR required by the system employing the linear MMSE detector diverges rapidly escalates upon increasing $L_{s}$. On the other hand, the SNR required by the SOPHIE-aided system increases at a moderate rate upon increasing the system load. Finally, the SNR requirements imposed by the SOPHIE and Log-MAP detectors are nearly identical.

\section{CONCLUSION}

An efficient DL-SDMA communication system employing the novel SOPHIE space-time processing method was investigated. We have demonstrated that the DL-SDMA system employing the SOPHIE detector proposed is capable of achieving the near optimum Log-MAP performance in overloaded scenarios, where the number of transmit antennas exceeds that of the receive antennas. Our future research will consider the employment of multilevel modulation schemes protected by Iteratively Detected Bit Interleaved Coded Modulation (BICM-ID) and Turbo Trellis Coded Modulation (TTCM) [6].

\section{REFERENCES}

[1] S. Ng and L. Hanzo, "On the MIMO channel capacity of multidimensional signal sets," Vehicular Technology Conference, 2004. VTC2004-Fall. 2004 IEEE 60th, vol. 3, pp. 1594 - 1598, 2004.

[2] D. Tse, P. Viswanath, and L. Zheng, "Diversity-multiplexing tradeoff in multiple-access channels," IEEE Transactions on Information Theory, vol. 50, pp. 1859- 1874, 2004.

[3] L. Hanzo and M. Münster and B-J. Choi and T. Keller, OFDM and MC-CDMA for broadband multi-user communications, WLANs and Broadcasting. John Wiley and IEEE press, 2003.

[4] P. Vandenameele, L. van der Perre, and M. Engels, Space division multiple access for wireless local area networks. Kluwer, 2001.

[5] J. Blogh and L. Hanzo, 3G systems and intelligent networking. John Wiley and IEEE Press, 2002, (For detailed contents, please refer to http://www-mobile.ecs.soton.ac.uk.).

[6] L. Hanzo, T. Liew, and B. Yeap, Turbo coding, turbo equalisation and space-time coding. John Wiley and IEEE Press, 2002, (For detailed contents, please refer to http://www-mobile.ecs.soton.ac.uk.).

[7] R. Irmer, "Multiuser transmission in code division multiple access mobile communications systems," Ph.D. dissertation, Technische Universitat Dresden, 2005.

[8] L.-U. Choi and R. Murch, "A transmit preprocessing technique for multiuser MIMO systems using a decomposition approach," IEEE Transactions on Wireless Communications, vol. 3, no. 1, pp. 20 - 24, 2004.

[9] Q. Spencer, A. Swindlehurst, and M. Haardt, "Zero-forcing methods for downlink spatial multiplexing in multiuser MIMO channels," IEEE Transactions on Signal Processing, vol. 52, pp. 461 - 471, 2004.

[10] J. Akhtman and L. Hanzo, "Reduced-complexity maximum-likelihood detection in multiple-antenna-aided multicarrier systems," in Proceedings of the 5th International Workshop on Multi-Carrier Spread Spectrum, Oberpfaffenhofen, Germany, 14-16 September 2005, to be published September 2005.

[11] — " "Novel optimized-hierarchy RSA-aided space-time processing method," University of Southampton, Tech. Rep., May 2005, mobile VCE Core 3 Programme - Wireless Enablers 2.2.

[12] D. Pham, K. Pattipati, P. Willet, and J. Luo, "An improved complex sphere decoder for v-blast systems," IEEE Signal Processing Letters, vol. 11, pp. 748-751, 2004.

[13] J. E. Gentle, Numerical linear algebra for applications in statistics. Berlin: Springer-Verlag, 1998.

[14] J. Ha, A. N. Mody, J. H. Sung, J. R. Barry, S. W. McLaughlin, and G. L. Stuber, "LDPC coded OFDM with Alamouti/SVD diversity technique," Wireless Personal Communications, vol. 23, pp. 183-194, 2002. 